\title{
A Novel QoS Multicast Model in Mobile Ad Hoc Networks
}

\author{
Guojun Wang ${ }^{1,2}$, Jiannong $\mathrm{Cao}^{1}$, \\ ${ }^{1}$ Department of Computing \\ Hong Kong Polytechnic University \\ Hung Hom, Kowloon, Hong Kong \\ \{csgjwang,csjcao,cskcchan\}@comp.polyu.edu.hk
}

\author{
Lifan Zhang ${ }^{2}, \quad$ Keith C. C. Chan ${ }^{1}$ \\ ${ }^{2}$ School of Information Science and Engineering \\ Central South University \\ Changsha, Hunan Province, P. R. China, 410083 \\ \{csgjwang@csu.edu.cn,fan_stars@126.com\}
}

\author{
$\mathrm{Jie} \mathrm{Wu}^{3}$ \\ ${ }^{3}$ Department of Computer Science and Engineering \\ Florida Atlantic University \\ Boca Raton, FL 33431 \\ jie@cse.fau.edu
}

\begin{abstract}
Multicast applications for large-scale Mobile Ad hoc NETworks (MANETS) require an efficient and effective Quality of Service (QoS)-aware multicast model. The new requirements to guarantee QoS are high availability and good load balancing due to limited bandwidth and transmission power of Mobile Nodes (MNs). We propose a logical Hypercube-based Virtual Dynamic Backbone (HVDB) model for QoS-aware multicast communications. In this model, high fault tolerance and small diameter of hypercubes are the basis for high availability, and regularity and symmetry of hypercubes contribute to good load balancing. Based on this model, we design proactive local logical route maintenance, summary-based membership update, and logical location-based multicast routing algorithms in large-scale MANETs.
\end{abstract}

\section{Introduction}

Ad hoc networks are self-organizing, rapidly deployable, and dynamically reconfigurable networks, which require no fixed infrastructure. Ad hoc networks in which the nodes are connected by wireless links and can be mobile are referred to as MANETs, where all the MNs function as hosts and routers at the same time. Two MNs communicate directly if they are within the radio transmission range of each other. Otherwise, they reach each other via a multi-hop route.

Many existing and forthcoming applications in MANETs require the collaboration of groups of mobile users. Communications in battlefield and disaster relief scenarios, video conferencing and multi-party gaming in conference room or classroom settings, and emergency warnings in vehicular networks are example applications. As a consequence, multicast in MANETs becomes a hot research topic in recent years. Multicast is a communication scheme for sending the same messages from a source to a group of destinations. MANETs are inherently ready for multicast communications due to their broadcast nature. However, limited bandwidth between MNs and highly dynamic topology due to unpredictable node mobility make the design of scalable and QoS-aware multicast routing protocols much more complicated than that in the traditional networks.

Most research works focus on small or medium-scale MANETs up to several hundreds of MNs proposed by the IETF MANET Working Group [15]. In recent years, some research works focus on large-scale MANETs with thousands, even hundreds of thousands of MNs, e.g., the landmark routing with mobile backbones for digitized battlefield [30], the CarNet system [18], the Terminodes system [2], and the Ad Hoc City [10] for metropolitan environment.

As MANETs are infrastructure-less, many virtual backbone-based routing schemes have been proposed to seek for similar capabilities of the high speed and broadband backbone in the Internet in supporting efficient data transportation. In the literature, two major techniques are used to construct a virtual backbone, i.e., connected dominating set [26, 29] and clustering [30, 23].

Because the search space for route discovery is reduced to the nodes in the virtual backbone consisting of the dominating set or the Cluster Heads (CHs), routing based on the virtual backbone scales better than that based on flat 
MANETs. However, the virtual backbone-based routing protocols still cannot scale well in large-scale MANETs when the number of nodes in the backbone becomes large.

In theory, a multi-tier hierarchy can potentially solve the scalability problem in the two-tier hierarchy. Therefore, a natural way is to further organize the backbone nodes into multiple tiers in large-scale MANETs. However, this scalability is not automatically guaranteed if too many tiers exist in the hierarchy. (1) Due to the mobility and failure of nodes, all the backbone nodes may join or leave the hierarchy at any time, which makes the maintenance of multitier routing tables quite challenging. (2) Most traffic load goes through the nodes in the higher tiers of the hierarchy, and these nodes become the bottlenecks. (3) There are some hardware limitations, e.g., different types of radio capabilities are required at different tiers. Although multiple radios in some backbone nodes are common practice in military applications, they may not be practical in many commercial applications if too many tiers of radios are required. Due to these reasons, one generally uses a backbone with only a few tiers (say, two) [30].

In order to solve the scalability problem in large-scale MANETs, researchers have developed many location-based routing protocols. Recent surveys on these protocols can be found in [16, 27]. In location-based routing, each node determines its own location through the use of Global Positioning System (GPS) or some other type of positioning service. A location service is used by the sender of a packet to determine the location of the destination and to include it in the header of the packet. The routing decision at each forwarding node, is then based on the locations of the forwarding node's neighbors and the destination node. In this way, the location-based routing need not to maintain routing tables. Therefore, location-based routing can scale quite well in large-scale MANETs.

In this paper, we propose a novel logical Hypercubebased Virtual Dynamic Backbone (HVDB) model using location information to support QoS multicast in MANETs. The proposed model is derived from $n$-dimensional hypercubes, which have many desirable properties, such as high fault tolerance, small diameter, regularity, and symmetry. Due to these properties, the proposed model meets the new QoS requirements of high availability and good load balancing.

This model uses the mobility prediction and locationbased clustering technique in [23] to form stable clusters, which elects an $\mathrm{MN}$ as a $\mathrm{CH}$ when it satisfies the following criteria: (1) it has the highest probability, in comparison to other MNs within the same cluster, to stay for longer time within the cluster; (2) it has the minimum distance from the center of the cluster. Based on this technique, this model further abstracts a flat structure into three tiers: the mobile node tier, the hypercube tier, and the mesh tier, where each $\mathrm{CH}$ elected by their clustering algorithm can be simply mapped to a hypercube node at the hypercube tier.

The remainder of the paper is organized as follows. We present some related works in Section 2. The proposed model is introduced in Section 3. Section 4 describes the design of our HVDB-based multicast routing protocol. Section 5 concludes this paper.

\section{Related Works}

\subsection{Preliminaries of Hypercubes}

An $n$-dimensional hypercube has $2^{n}$ nodes. Each node is labelled by a bit string $k_{1} \cdots k_{n}\left(k_{i} \in\{0,1\}, 1 \leq i \leq n\right)$. Two nodes are connected by a link if and only if their labels differ by exactly one bit. The Hamming distance between two nodes $u$ and $v$, denoted by $H(u, v)$, is the number of bits in which $u$ and $v$ differ.

An $n$-dimensional hypercube has many desirable properties: (1) High fault tolerance: The hypercube offers $n$ node disjoint paths between each pair of nodes, therefore it can sustain up to $n$ - 1 node failures; (2) Small diameter: The diameter of the hypercube is defined as the maximal Hamming distance between any pair of nodes in the hypercube, which is $n$; (3) Regularity: The hypercube has a very regular structure, in which every node plays exactly the same role, and no node is more loaded than any others to achieve load balancing; (4) Symmetry: The hypercube is symmetrical in graph terminology. In particular, any $(k+1)$-dimensional subcube in the hypercube consists of two $k$-dimensional subcubes for all $1 \leq k<n$, each of which is also symmetrical.

The hypercube is used to be a very hot research topic. It is originally proposed as an efficient interconnection network topology for Massively Parallel Processors (MPPs). In recent years, much research has been done to apply the hypercube to other network environments, such as multicast communications in the Internet $[14,7]$, hypercube-like prefix routing in $\mathrm{P} 2 \mathrm{P}$ networks $[24,19,31]$, and hypercubebased overlay formation for $\mathrm{P} 2 \mathrm{P}$ computing [20].

In [12], the authors propose the incomplete hypercube, which may contain any number of nodes. We generalize the incomplete hypercube by allowing any number of nodes/links to be absent due to many reasons such as mobility, transmission range, and failure of nodes.

\subsection{Location-based Multicast Routing}

Traditional unicast routing protocols designed for flat MANETs and hierarchical extensions, cannot scale well in large-scale MANETs. Similarly, traditional multicast routing protocols, e.g., flooding-based, tree-based, and meshbased, cannot scale well in large-scale MANETs either. 
In recent years, location-based unicast routing has attracted much attention because it scales quite well in largescale MANETs. Accordingly, researchers have proposed to use location information in multicast routing protocols. In the Dynamic Source Multicast (DSM) protocol [1], when a packet is to be multicast, the sender first locally computes a snapshot of the global network topology according to the location and transmission radius information collected from all the nodes in the network. A multicast tree for the addressed multicast group is then computed locally based on the snapshot. The resulting multicast tree is then optimally encoded and is included in the packet header. This protocol improves the scalability because it eliminates the maintenance of the multicast session state in each router, which has to be done in traditional multicast tree or multicast meshbased protocols. However, its scalability is still limited because the location and transmission radius information has to be periodically broadcast from each node to all the other nodes in the network.

In [6], the Small Group Multicast (SGM) protocol based on packet encapsulation is proposed. This protocol builds an overlay multicast packet distribution tree on top of the underlying unicast routing protocol. Different from the DSM protocol that computes the multicast tree at each sender, this protocol constructs the tree in a distributed way: each node only constructs its out-going branches to the next-level subtrees and forwards the packet to the roots of the subtrees. This process repeats until all the destinations have been reached. This protocol is more scalable than the DSM protocol because the nodes in a group need not to know the global network topology. Instead, they are only aware of each other in terms of the group membership and the location information of the group nodes. However, this protocol does not specify a method for dynamic joins and leaves in terms of location update among the group nodes. Therefore, this protocol is more suitable for the groups in which the group membership is static.

In [17], the Position-Based Multicast (PBM) protocol is proposed using only locally available location information about the destination nodes. This protocol provides a solution in order to approximate the optima for two potentially conflicting properties of the multicast distribution tree: (1) the length of the paths to the individual destinations should be minimal, and (2) the total number of hops needed to forward the packet to all the destinations should be as small as possible. If not properly handled, a greedy multicast forwarding may lead to a problem when a packet arrives at a node that does not have any neighbor providing progress for one or more destinations. This problem is solved in location-based unicast routing, such as using the right hand rule-based recovery strategy in [11]. This protocol extends the strategy to support the packet with multiple destinations. This protocol can deal with group mem- bers distributed in large-scale MANETs. However, it cannot scale well in terms of the number of group nodes due to the fact that the location and group membership information is required at each sender of the multicast group.

In [28], the Scalable Position-Based Multicast (SPBM) protocol is proposed to extend PBM. SPBM uses a hierarchical aggregation of membership information: the further away a region is from an intermediate node, the higher the level of aggregation should be for this region. This hierarchical scheme improves scalability. However, because all the nodes in the network are involved in the membership update, it still cannot scale well in large-scale MANETs. In this paper, we solve this problem by summarizing the group membership information in a novel way and disseminating this information to only a portion of nodes in the network. Therefore, our scheme can potentially scale well in terms of both the number of groups and the number of group nodes in each group in large-scale MANETs.

\subsection{QoS-aware Routing Issues}

Generally speaking, QoS is a loosely defined term. There are some metrics affecting QoS, such as delay, bandwidth, packet loss, and energy consumption. QoS-aware routing has been studied extensively in the wired networks such as the Internet. Due to the node mobility and the scarcity of resources such as energy of nodes and bandwidth of wireless links, it is much more difficult to provide QoS guarantee in MANETs than in the Internet. In fact, guaranteeing QoS in such a network may be impossible if the nodes are too mobile [3]. In the literature, there are only a few works tackling this problem in MANETs.

In [5], a hard-QoS protocol based on the well-known IntServ model is proposed in MANETs, which searches multiple paths in parallel in order to find the most qualified one. In [25], the authors propose to use location information in QoS routing decisions, and consider connection time (estimated lifetime of a link) as a QoS constraint. In [9], the authors present a protocol for TDMA-based bandwidth reservation for QoS routing in MANETs. It solves the race condition and parallel reservation problems by maintaining three-state information (free/allocated/reserved) at each MN.

In [13], a soft-QoS protocol based on the well-known Differentiated Services model is proposed in MANETs. It extends the Dynamic Source Routing (DSR) protocol to embed the QoS constraints in the discovery, maintenance of routes, and the traffic management. In highly dynamic MANETs, soft-QoS protocols may have better overall performance than hard-QoS protocols due to the highly unpredictable topological change of the MANETs.

In MANETs, network nodes/links may be broken sometimes, disrupting the continuity of an on-going session and 
potentially terminating the session, thus inducing the QoS problem. Many papers view the QoS as a scheme in providing fault tolerance $[4,8]$. In particular, in [22], the authors propose to pre-compute some routes before existing routes break and thus avoid route re-computation delay. In this sense, the HVDB model proposed in this paper helps to provide fault tolerance due to the high fault tolerance of hypercubes.

The QoS problem is hard to tackle even in the wired network. In [21], the authors point out that high availability and even distribution of traffic over the network are a prerequisite for the economical provisioning of QoS. We complement that it is especially true in MANETs due to limited bandwidth and energy of MNs. Here high availability indicates that a network has the capability of hiding or quickly responding to faults, making users no sense of faults in the network; Load balancing indicates that traffic load be distributed evenly in the network to the greatest extent in order to eliminate hot spots in the network. Based on these, traditional QoS models, such as IntServ and DiffServ models, can perform much more effectively in MANETs.

\section{The QoS Multicast Model}

In this section we introduce our logical Hypercube-based Virtual Dynamic Backbone (HVDB) model shown in Figure 1, which has high availability and good load balancing properties in large-scale MANETs.

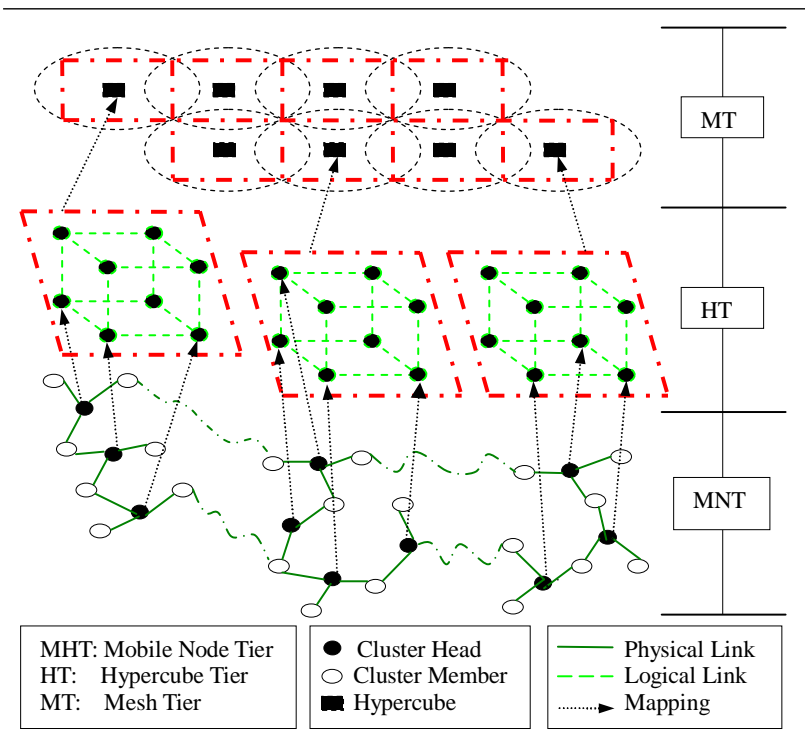

Figure 1. The HVDB Model

The Mobile Node Tier (MNT) consists of MNs that move in and out of reach to each other with regard to the radio propagation range. The MNs are grouped into clus- ters according to some criteria. Every cluster has one $\mathrm{CH}$ and multiple cluster members. These $\mathrm{CHs}$ are responsible for forwarding packets and communicating between clusters, and managing their cluster members. In this tier, we use the same way to divide a geographical area (or even the whole earth) into equal regions of circular shape as in [23]. Each MN can determine the circle where it resides if location information is available. We call the circle a Virtual Circle (VC), accordingly the center of the VC is called Virtual Circle Center (VCC). If there is a $\mathrm{CH}$ in a VC, then we view the $\mathrm{VCC}$ as the $\mathrm{CH}$; if not, then it is only a placeholder. We also use the mobility prediction and location-based clustering technique in [23], which has been shown to be able to form clusters much more stably than other schemes.

The Hypercube Tier (HT) comprises multiple logical $k$ dimensional hypercubes ( $k$ is relatively small in our consideration, e.g., 3, 4, 5, or 6), whose nodes are actually the CHs. A logical hypercube node becomes an actual one only when a $\mathrm{CH}$ exists in the VC. There is a one-to-one mapping relation between a $\mathrm{CH}$ and a hypercube node. The $\mathrm{CHs}$ located within a predefined region build up a logical $k$-dimensional hypercube, which is probably an incomplete hypercube. The hypercube is logical in the sense that the logical link between two adjacent logical hypercube nodes possibly consists of multi-hop physical links.

The Mesh Tier (MT) is a logical 2-dimensional mesh network by viewing each $k$-dimensional hypercube as one mesh node. In the same way, the 2-dimensional mesh is possibly an incomplete mesh, and the link between two adjacent mesh nodes is logical and physically multi-hop. A mesh node becomes an actual mesh node only when a logical hypercube exists in it.

In Figure 1, the mesh tier is drawn in circle regions, and the hypercube tier isn't drawn with circles for clarity. The higher two tiers comprise the HVDB. In particular, the HVDB has the non-virtual and non-dynamic properties, which are similar to reality and stability properties of the backbone in the Internet, respectively.

In order to realize the non-virtual property of the HVDB, we assume each $\mathrm{MN}$ can acquire its location information such as geographical position, moving velocity, and moving direction, using some devices such as a GPS. Then each $\mathrm{MN}$ can determine the VC where it resides. Each VC represents a node in the HVDB. If at least one $\mathrm{MN}$ capable of functioning as a $\mathrm{CH}$ exists in a $\mathrm{VC}$, then there is an actual node in the HVDB. These VCs are overlapped with each other and an $\mathrm{MN}$ within the overlapped regions can be a cluster member of two or multiple clusters at the same time for more reliable communications.

In order to realize the non-dynamic property of the HVDB, we mitigate the dynamic behavior of MANETs by making two assumptions. (1) We assume to use the clustering technique in [23]. (2) We assume MNs have dif- 
ferent computation and communications capabilities, with the $\mathrm{CHs}$ having stronger capability than others. The former guarantees to form stable clusters in large-scale MANETs. The latter guarantees to form a stable HVDB. We argue the latter is reasonable in practice and easy to realize, e.g., in a battlefield, a mobile device equipped on a tank can have stronger capability than the one equipped for a foot soldier.

\section{The QoS-aware Multicast Protocol}

Based on the HVDB model, we describe the design of the proactive local logical route maintenance algorithm, the summary-based membership update algorithm, and the logical location-based multicast routing algorithm.

\subsection{Proactive Local Logical Route Maintenance}

Many traditional routing protocols cannot scale well in large-scale MANETs because global topology has to be known either by all the MNs for proactive protocols or by the senders for reactive protocols. In order to scale well in large-scale MANETs, our protocol requires only the $\mathrm{CHs}$ to maintain local topology in a distributed way. In this subsection, we show how to proactively maintain local logical routes at each $\mathrm{CH}$ in our HVDB model.

In our model, the whole network is divided into many VCs of equal size. The VCs are grouped to form logical hypercubes. We consider logical hypercubes with small dimension, which is set as a system parameter. A simple function is used to map each $\mathrm{CH}$ to a hypercube node, using system parameters such as central coordinate, length and width of the whole network, diameter of VCs, and dimension of logical hypercubes. We define four kinds of logical identifiers: Cluster Head ID (CHID), Hypercube Node ID (HNID), Hypercube ID (HID), and Mesh Node ID (MNID). The relation between CHID and HNID is one-to-one mapping, the relation between HNID and HID is many-to-one mapping, and the relation between HID and MNID is oneto-one mapping. In this paper, the logical identifier of each logical node is also called logical location.

Figure 2 shows an example MANET with $8 * 8 \mathrm{VCs}$, which is further divided into four 4-dimensional logical hypercubes. One logical hypercube is shown in Figure 3, with additional logical links connecting some VCs according to the logical relationship among these VCs.

The $\mathrm{CHs}$ are classified into Border Cluster Heads (BCHs) and Inner Cluster Heads (ICHs). A BCH is a $\mathrm{CH}$ that may have logical link between two adjacent mesh nodes, i.e., two adjacent logical hypercubes. A BCH forwards traffic among logical hypercubes, while an ICH does that within a logical hypercube.

We define the number of logical hops of a logical link as follows. If a logical link satisfies two conditions, (1) it

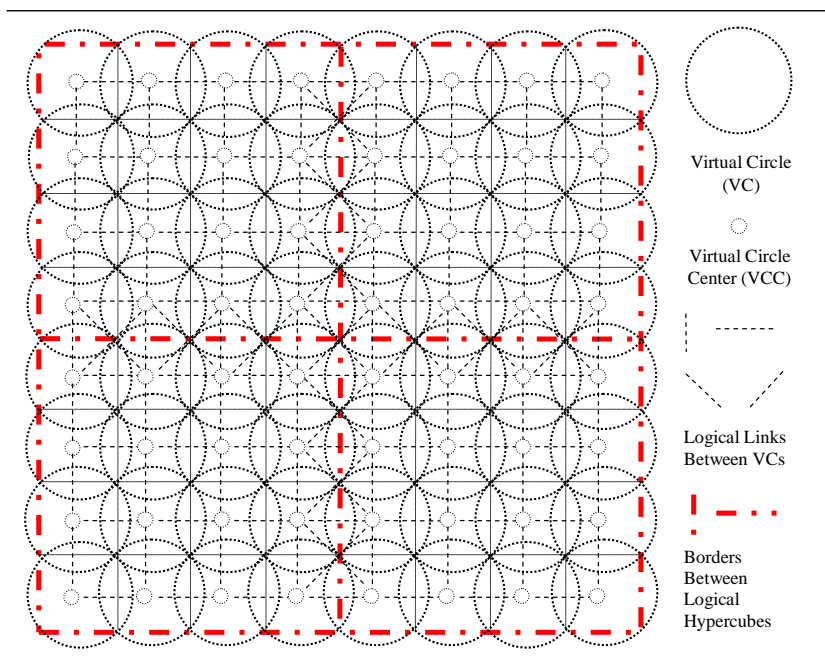

Figure 2. An Example MANET with $8 \star 8$ VCs

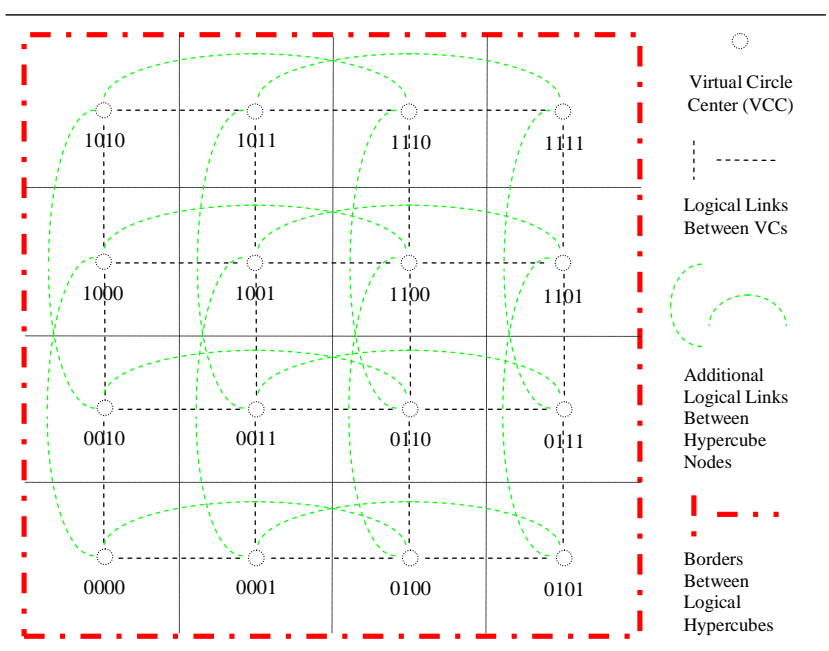

Figure 3. A 4-Dimensional Logical Hypercube

connects two $\mathrm{CHs}$, (2) it does not rely on any other $\mathrm{CH}$ to route packets along the link, then the number of logical hops of the logical link is 1 , and the logical link is called a 1 logical hop route. Accordingly, the number of logical hops of a logical link connecting any two $\mathrm{CHs}$ is the total number of concatenated 1-logical hop routes that comprise the logical link between the two CHs. For example, the number of logical hops that comprise 1-logical hop routes of $1000 \rightarrow 1100 \rightarrow 1101$ is 2 .

Our proactive local logical route maintenance algorithm is shown in Figure 4. Each $\mathrm{CH}$ periodically exchanges its local logical route information with those $\mathrm{CHs}$ that are at most $k \geq 1$ logical hops away. Here $k$ is a system parameter, e.g., $k=4$. In particular, the information such as delay and bandwidth is maintained in each specific local logi- 


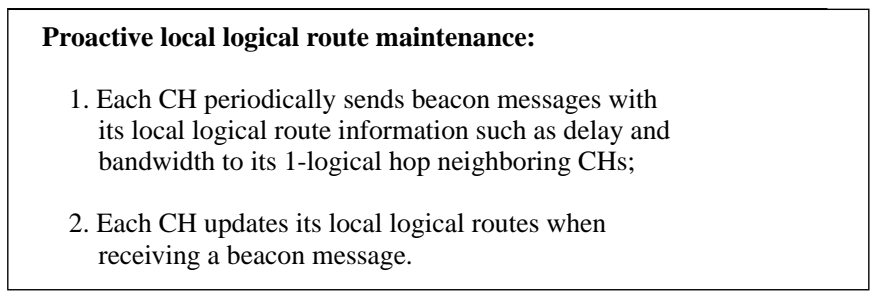

Figure 4. The Proactive Local Logical Route Maintenance Algorithm

cal route, which is used for QoS routing.

Finally we show some local logical routes maintained by the algorithm at the hypercube node labelled with 1000 . The 1-logical hop routes include: $1000 \rightarrow 1001,1000 \rightarrow$ $1010,1000 \rightarrow 0010,1000 \rightarrow 1100,1000 \rightarrow 0000$, and some route(s) to its adjacent logical hypercube(s). The 2logical hop routes include: $1000 \rightarrow 1001 \rightarrow 1100,1000 \rightarrow$ $1100 \rightarrow 1101,1000 \rightarrow 0010 \rightarrow 0011,1000 \rightarrow 0010 \rightarrow$ 0110 , and many others.

\subsection{Summary-based Membership Update}

Based on the HVDB model, we propose to summarize the group membership information at three tiers. At the mobile node tier, each node in each cluster knows which multicast groups it has currently joined, which is called Local-Membership. Each MN periodically sends LocalMembership to its $\mathrm{CH}$. Each $\mathrm{CH}$ then summarizes the group membership according to all the information got from all its cluster members, which is called MNT-Summary.

At the hypercube tier, each hypercube node, i.e., each $\mathrm{CH}$ in the hypercube, periodically sends MNT-Summary to all the $\mathrm{CHs}$ within the hypercube. Each $\mathrm{CH}$ then summarizes the group membership information according to all the information got from all the $\mathrm{CHs}$ within the hypercube, which is called HT-Summary.

If we neglect the delay for transmitting the LocalMembership and MNT-Summary messages, then each $\mathrm{CH}$ in a logical hypercube has the same HT-Summary information. Therefore, any one of the $\mathrm{CHs}$ can be designated to periodically broadcast the HT-Summary information to all the $\mathrm{CHs}$ in the whole network. There are many ways to choose one to do such a task. The simplest way is to always designate the same $\mathrm{CH}$ to do the task. However, it may be not reliable, and the $\mathrm{CH}$ may become the bottleneck. Another way is to request each $\mathrm{CH}$ to be responsible for this task one by one. It eliminates the above two problems, but it may incur too much overhead in order to coordinate among the $\mathrm{CHs}$.

We propose a solution to reduce the overhead. Each $\mathrm{CH}$ uses its up-to-date information about its own MNTSummary and the collected MNT-Summary messages to de-

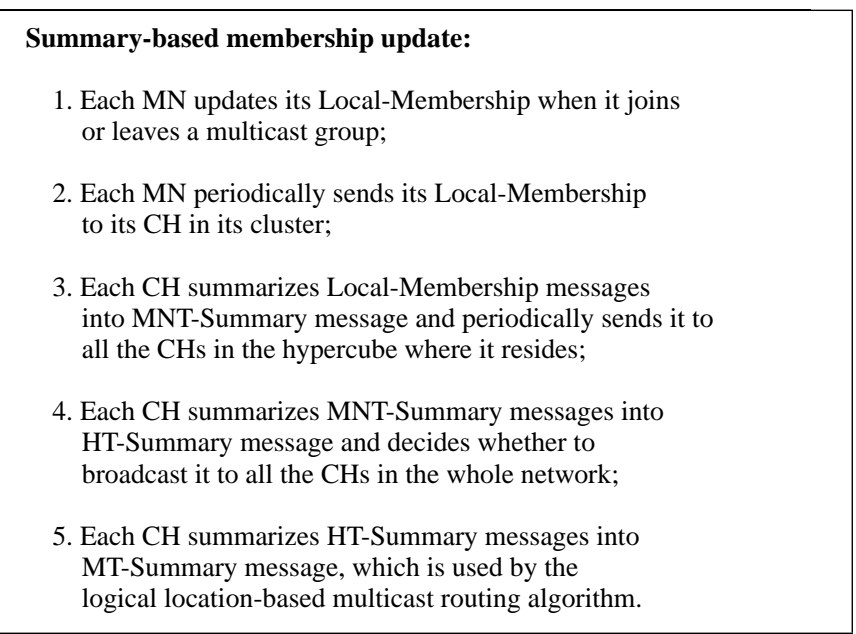

Figure 5. The Summary-based Membership Update Algorithm

cide whether itself will be responsible for doing the task or not according to some criteria. One criterion is to choose the $\mathrm{CH}$ that contains the largest number of multicast groups, or the largest number of group members. Another criterion is to choose the $\mathrm{CH}$ such that the total number of multicast groups, or the total number of group members, contained by itself and all its 1-logical hop neighboring $\mathrm{CHs}$, is the largest one. When considering the delay for transmitting membership messages, we argue the latter criterion can work well because the probability for only one $\mathrm{CH}$ satisfying the same criterion will be very high in most of the time.

At the mesh tier, each mesh node, in fact, all the $\mathrm{CHs}$ in the network, summarizes the HT-Summary messages into its MT-Summary membership information. Since each $\mathrm{CH}$ in the network only needs to know which logical hypercubes contain which groups of members, the timeout interval for broadcasting HT-Summary messages can be set much more larger than that for sending MNT-Summary or Local-Membership messages. Therefore, we argue that the proposed algorithm doesn't incur too much overhead.

Finally we present the summary-based membership update algorithm in Figure 5.

\subsection{Logical Location-based Multicast Routing}

In our membership scheme, each $\mathrm{CH}$ maintains highly summarized membership information about all the groups in the network. In particular, the MT-Summary information maintained by all the CHs becomes identical if the group membership doesn't change too drastically.

Based on this membership scheme, our multicast routing scheme is as follows. If an MN needs to send multicast messages to a specific group, it sends them to its $\mathrm{CH}$. Then the $\mathrm{CH}$ checks its MT-Summary to determine which logi- 
cal hypercubes contain members of this group, and the logical identifiers, i.e., the logical locations of these logical hypercubes are used to compute a multicast tree for the messages. The multicast tree is then cached for future use. Then the information about the multicast tree is encapsulated into the messages. Finally the messages are forwarded along the multicast tree.

The multicast tree is built at the mesh tier, and each node in the tree is a mesh node, i.e., a logical hypercube. In the multicast tree, we assume to use some location-based unicast routing algorithm to send a packet from one logical hypercube to its next hop logical hypercube. When a packet enters a logical hypercube at a certain logical node at its first time, two tasks are executed at the hypercube node: (1) the packet is re-encapsulated (possibly duplicated), then it is unicast to its next hop logical hypercube(s); (2) within the logical hypercube, the packet is forwarded to those hypercube nodes that contain group members.

In order to forward the packet within the logical hypercube, the hypercube node computes a multicast tree using its HT-Summary. The multicast tree is cached at the hypercube node for future use. The multicast tree is then encapsulated into the packet header in order to forward the packet within the logical hypercube. The packet forwarding using the multicast tree at the hypercube tier is different from that using the multicast tree at the mesh tier. In each logical hypercube, each hypercube node has already maintained all the local logical routes in advance which are at most $k$ logical hops away. If all the local logical routes at the multicast tree are at most $k$ logical hops away, then the packet forwarding can directly use them. In some extreme cases, e.g., if $k$ is too small but the logical hypercube is too large, then it is possible for some of the local logical routes at the multicast tree to go beyond $k$ logical hops away. In this paper, we suppose $k$ is sufficiently large and the hypercube is relatively small in order to avoid such extreme cases.

Besides the multicast routing at the mesh tier and the hypercube tier, it is also needed to route a packet to the group members (if exist) at the mobile node tier when a $\mathrm{CH}$, i.e., a hypercube node, receives the packet. Many methods such as local broadcast, can be used to route a packet to the group members within a cluster.

Finally we show our logical location-based multicast routing algorithm in Figure 6.

\section{Conclusions}

We have proposed a novel HVDB model to support QoS-aware multicast in large-scale MANETs. The proposed model is derived from $n$-dimensional hypercubes, which have many desirable properties, such as high fault tolerance, small diameter, regularity, and symmetry. The proposed model uses the location information of MNs and

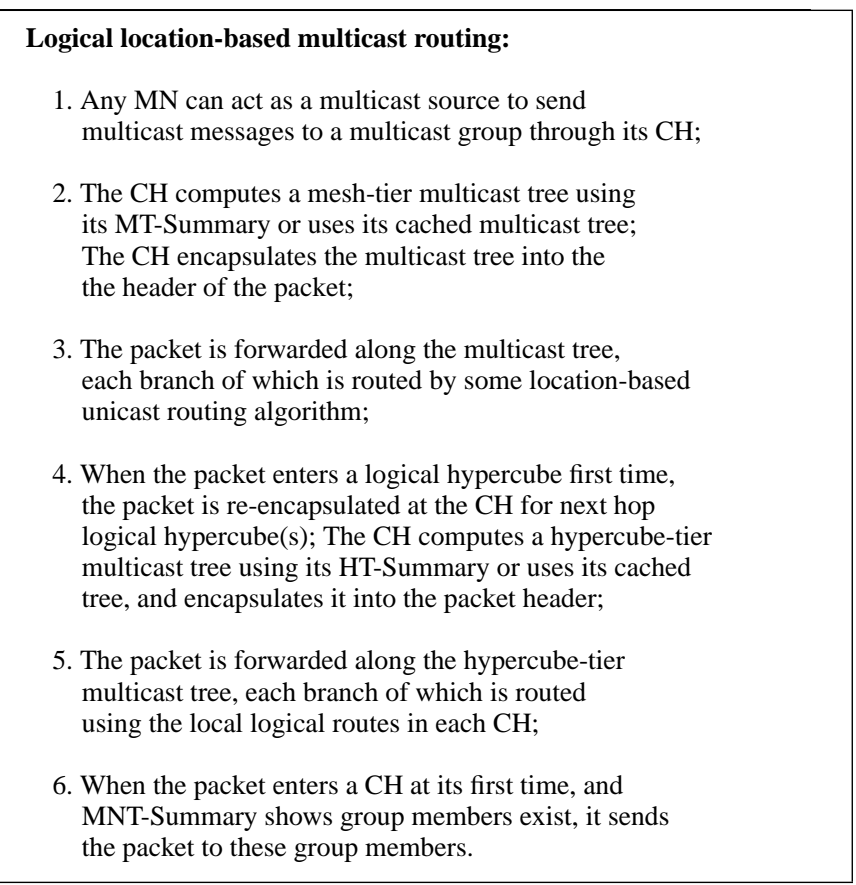

Figure 6. The Logical Location-based Multicast Routing Algorithm

meets the new QoS requirements: high availability and good load balancing. Firstly, in an incomplete logical hypercube, there are multiple disjoint local logical routes between each pair of $\mathrm{CHs}$, the high fault tolerance property provides multiple choices for QoS routing. That is, if the current logical route is broken, multiple candidate logical routes become available immediately to sustain the service without QoS being degraded. Secondly, small diameter facilitates small number of logical hops on the logical routes. Thirdly, due to the regularity and symmetry properties of hypercubes, no leader is needed in a logical hypercube, and every node plays almost the same role except for the slightly different roles of BCHs and ICHs. Thus, no single node is more loaded than any other nodes, and no problem of bottlenecks exists, which is likely to occur in tree-based architectures.

\section{Acknowledgment}

This work was supported by the Hong Kong Polytechnic University Central Research Grant G-YY41, the University Grant Council of Hong Kong under the CERG Grant PolyU 5170/03E, and the China Postdoctoral Science Foundation (No. 2003033472).

\section{References}

[1] S. Basagni, I. Chlamtac, V.R. Syrotiuk, "Location aware, dependable multicast for mobile ad hoc networks," Computer 
Networks (Elsevier), Vol. 36, pp. 659-670, 2001.

[2] L. Blazevic, S. Giordano, J.-Y. Le Boudec, "Self organized terminode routing," Cluster Computing (Kluwer Academics), Vol. 5, pp. 205-218, 2002.

[3] S. Chakrabarti, A. Mishra, "QoS issues in ad hoc wireless networks," IEEE Communications Magazine, Vol. 39, Issue 2, pp. 142-148, Feb. 2001.

[4] T.-W. Chen, P. Krzyzanowski, M.R. Lyu, C. Sreenan, J.A. Trotter, "Renegotiable quality of service-a new scheme for fault tolerance in wireless networks," Proc. 27th Annual International Symposium on Fault-Tolerant Computing (FTCS27), pp. 21-30, Jun. 1997.

[5] S. Chen, K. Nahrstedt, "Distributed quality-of-service routing in ad hoc networks," IEEE Journal on Selected Areas in Communications, Vol. 17, Issue 8, pp. 1488-1505, Aug. 1999.

[6] K. Chen, K. Nahrstedt, "Effective location-guided tree construction algorithms for small group multicast in MANET," Proc. IEEE 21th Annual Joint Conference of the IEEE Computer and Communications Societies (INFOCOM 2002), Vol. 3, pp. 1180-1189, Jun. 2002.

[7] R. Friedman, S. Manor, K. Guo, "Scalable stability detection using logical hypercube," IEEE Transactions on Parallel and Distributed Systems, Vol. 13, Issue 9, pp. 972-984, Sept. 2002.

[8] S.S. Gokhale, S.K. Tripathi, "Effect of unreliable nodes on QoS routing," Proc. 7th International Conference on Network Protocols (ICNP 1999), pp. 173-181, Oct.-Nov. 1999.

[9] I. Jawhar, J. Wu, "A race-free bandwidth reservation protocol for QoS routing in mobile ad hoc networks," Proc. 37th Annual Hawaii International Conference on System Sciences, pp. 293-302, Jan. 2004.

[10] J.G. Jetcheva, Y.-C. Hu, S. PalChaudhuri, A.K. Saha, D.B. Johnson, "Design and evaluation of a metropolitan area multitier wireless ad hoc network architecture," Proc. 5th IEEE Workshop on Mobile Computing Systems and Applications (WMCSA2003), pp. 32-43, Oct. 2003.

[11] B. Karp, H.T. Kung, "GPSR: Greedy Perimeter Stateless Routing for wireless networks," Proc. 6th annual international conference on Mobile computing and networking (MobiCom 2000), pp. 243-254, Aug. 2000.

[12] H. P. Katseff, "Incomplete hypercubes," IEEE Transactions on Computers, Vol. 37, No. 5, pp. 604-607, May 1988.

[13] H. Labiod, A. Quidelleur, "QoS-ASR: An adaptive source routing protocol with QoS support in multihop mobile wireless networks," Proc. IEEE 56th Vehicular Technology Conference (VTC 2002-Fall), Vol. 4, pp. 1978-1982, Sept. 2002.

[14] J. Liebeherr, B.S. Sethi, "A scalable control topology for multicast communications," Proc. 17th Annual Joint Conference of the IEEE Computer and Communications Societies (INFOCOM 1998), Vol. 3, pp. 1197-1204, Mar.-Apr. 1998.

[15] Mobile Ad-hoc Networks (MANETs) Working Group, http://www.ietf.org/html.charters/manet-charter.html.

[16] M. Mauve, A. Widmer, H. Hartenstein, "A survey on position-based routing in mobile ad hoc networks," IEEE Network, Vol. 15, Issue 6, pp. 30-39, Nov.-Dec. 2001.

[17] M. Mauve, H. Fubler, J. Widmer, T. Lang, "MobiHoc Poster: Position-based multicast routing for mobile Ad-hoc networks," ACM SIGMOBILE Mobile Computing and Communications Review, Vol. 7, No. 3, pp. 53-55, Jul. 2003.
[18] R. Morris, J. Jannotti, F. Kaashoek, J. Li, D. Decouto, "CarNet: A scalable ad hoc wireless network system," Proc. 9th workshop on ACM SIGOPS European workshop: beyond the PC: new challenges for the operating system, pp. 61-65, 2000.

[19] A. Rowstron and P. Druschel, "Pastry: Scalable, distributed object location and routing for large-scale peer-to-peer systems," Proc. IFIP/ACM International Conference on Distributed Systems Platforms (Middleware), Heidelberg, Germany, pp. 329-350, Nov. 2001.

[20] M. Schlosser, M. Sintek, S. Decker, W. Nejdl, "A scalable and ontology-based P2P infrastructure for semantic Web services," Proc. Second International Conference on Peer-toPeer Computing (P2P 2002), pp. 104-111, Sept. 2002.

[21] G. Schollmier, C. Winkler, "Providing sustainable QoS in next-generation networks," IEEE Communications Magazine, Vol. 42, Issue 6, pp. 102-107, Jun. 2004.

[22] S.H. Shah, K. Nahrstedt, "Predictive location-based QoS routing in mobile ad hoc networks," Proc. IEEE International Conference on Communications (ICC 2002), Vol. 2, pp. 10221027, Apr.-May 2002.

[23] S. Sivavakeesar, G. Pavlou, A. Liotta, "Stable clustering through mobility prediction for large-scale multihop intelligent ad hoc networks," Proc. IEEE 2004 Wireless Communications and Networking Conference (WCNC 2004), Vol. 3, pp. 1488-1493, Mar. 2004.

[24] I. Stoica, R. Morris, D. Liben-Nowell, D.R. Karger, M.F. Kaashoek, F. Dabek, H. Balakrishnan, "Chord: A scalable peer-to-peer lookup protocol for Internet applications," IEEE/ACM Transactions on Networking, Vol. 11, Issue 1, pp. 17-32, Feb. 2003.

[25] I. Stojmenovic, M. Russell, B. Vukojevic, "Depth first search and location based localized routing and QoS routing in wireless networks," Proc. 2000 International Conference on Parallel Processing (ICPP 2000), pp. 173-180, Aug. 2000.

[26] I. Stojmenovic, M. Seddigh, J. Zunic, "Dominating sets and neighbor elimination-based broadcasting algorithms in wireless networks," IEEE Transactions on Parallel and Distributed Systems, Vol. 13, Issue 1, pp. 14-25, Jan. 2002.

[27] I. Stojmenovic, "Position-based routing in ad hoc networks," IEEE Communications Magazine, Vol. 40, Issue 7, pp. 128134, Jul. 2002.

[28] M. Transier, H. Fubler, J. Widmer, M. Mauve, W. Effelsberg, "Scalable position-based multicast for mobile ad-hoc networks," Proc. First International Workshop on Broadband Wireless Multimedia: Algorithms, Architectures and Applications (BroadWim 2004), San Jose, CA, Oct. 2004.

[29] J. Wu, "Extended dominating-set-based routing in ad hoc wireless networks with unidirectional links," IEEE Transactions on Parallel and Distributed Systems, Vol. 13, Issue 9, pp. 866-881, Sept. 2002.

[30] K. Xu, X. Hong, M. Gerla, "Landmark routing in ad hoc networks with mobile backbones," Journal of Parallel and Distributed Computing (Elsevier), Vol. 63, pp. 110-122, 2003.

[31] B.Y. Zhao, L. Huang, J. Stribling, S.C. Rhea, A.D. Joseph, J.D. Kubiatowicz, "Tapestry: A resilient global-scale overlay for service deployment," IEEE Journal on Selected Areas in Communications, Vol. 22, Issue 1, pp. 41-53, Jan. 2004. 